\title{
Prospective evaluation of selected cytokines for hydrocephalus
}

\section{surgery}

\author{
Christian Geyer*, Gerd Graefe, Anke Pauer, Katrin Bauer, Udo Rolle, \\ Roman Metzger, Holger Till and Ulrich Sack
}

\author{
Address: Klinik und Poliklinik fuer Kinderchirurgie, Universität Leipzig, Oststraße 21-25, 04317 Leipzig, Germany \\ Email: Christian Geyer* - christian.geyer@medizin.uni-leipzig.de \\ * Corresponding author
}

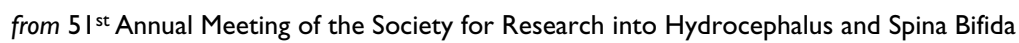 \\ Heidelberg, Germany. 27-30 June 2007 \\ Published: 20 December 2007 \\ Cerebrospinal Fluid Research 2007, 4(SuppI I):S46 doi:I0.1 186/1743-8454-4-SI-S46
}

This abstract is available from: http://www.cerebrospinalfluidresearch.com/content/4/SI/S46

(c) 2007 Geyer et al; licensee BioMed Central Ltd.

\section{Background}

The aim of the study was the evaluation of different cytokines in children with hydrocephalus (HC) of different genesis. Increasing ICP should lead to changes in cytokine levels in CSF and serum, respectively. We expected knowledge about the cellular pathology in HC children, but also findings improving diagnostics as well as early detection of higher risk for shunt blockage.

\section{Materials and methods}

From 2003 to 200647 children with HC receiving surgery for shunting and shunt revision were selected for cytokine analysis in CSF and serum. Interleukin-6 (IL-6), vascular endothelial growth factor (VEGF), insulin-like growth factor 1 (IGF-1), leptin and fibroblast growth factor (FGF) were detected in CSF and serum samples by commercially available ELISA kits (R\&D).

\section{Results}

All selected cytokines could be detected in the serum as well as in the CSF of all children. In comparison to children with HC caused from other geneses, children with posthaemorrhagic HC were characterised by significantly higher IL-6 levels in CSF. There was no correlation between ICP at time of operation and the level of cytokines in CSF and serum. In children with enhanced serum levels of FGF and IGF-1, a revision of the shunting system due to blockage was necessary within 18 months. In contrast to shunt revisions due to mechanical problems (dislocation, disconnection), shunt dysfunction due to obstruction was characterised by significantly higher levels of VEGF and leptin in CSF.

\section{Conclusion}

The initial assumption that cytokine levels in HC depend on the ICP was not confirmed. Depending on the clinical course we have found differentiated cytokine levels. The determined cytokines probably reflect complex cellular regulatory processes within the ventricular fluid that are grossly unknown. Our results have to be verified in a prospective clinical trial to determine the predictive value of cytokines for possible shunt complications. 